\title{
Cross-Coupling of Alkynylsilanols with Aryl Halides Promoted by Potassium Trimethylsilanolate
}

\author{
Scott E. Denmark* and Steven A. Tymonko \\ Roger Adams Laboratory, Department of Chemistry, University of Illinois, 600 South Mathews \\ Avenue, Urbana, Illinois, 61801

\section{SUPPORTING INFORMATION}

Table of Contents

Page

General Experimental

Response Factors

Procedure for Preparation of Alkynylsilanols

Competition Studies of Alkynylsilicon Compounds

Cross-Coupling of Dimethyl(1-heptynyl)silanol with Aryl Iodides

References

$S 26$

\section{General Experimental}

All reactions were performed in oven-dried $\left(140{ }^{\circ} \mathrm{C}\right)$ or flame-dried glassware under an inert atmosphere of dry argon. DME was distilled from $\mathrm{Na}$ and benzophenone. Brine refers to a saturated aqueous solution of $\mathrm{NaCl}$. Commercial reagents were purified by distillation or recrystallization prior to use.

Analytical thin-layer chromatography was performed on Merck silica or aluminum oxide, basic gel plates with QF-254 indicator. Visualization was accomplished with UV light and/or $\mathrm{KMnO}_{4}$ solution. Diethyl ether was of reagent grade and used as received; other solvents for chromatography and filtration were technical grade and distilled from the indicated drying agents: hexane and pentane $\left(\mathrm{CaCl}_{2}\right)$; ethyl acetate $\left(\mathrm{K}_{2} \mathrm{CO}_{3}\right)$. 
${ }^{1} \mathrm{H}$ NMR spectra and ${ }^{13} \mathrm{C}$ NMR spectra were recorded on $500 \mathrm{MHz},{ }^{1} \mathrm{H}\left(126 \mathrm{MHz},{ }^{13} \mathrm{C}\right)$ spectrometers. Spectra are referenced to residual chloroform $\left(\delta 7.26 \mathrm{ppm},{ }^{1} \mathrm{H} ; \delta 77.0 \mathrm{ppm},{ }^{13} \mathrm{C}\right)$ and residual benzene $\left(\delta 7.16 \mathrm{ppm},{ }^{1} \mathrm{H} ; \delta 128.0 \mathrm{ppm},{ }^{13} \mathrm{C}\right)$. Chemical shifts are reported in ppm $(\delta)$; multiplicities are indicated by s (singlet), d (doublet), t (triplet), q (quartet), m (multiplet) and br (broad). Coupling constants, $J$, are reported in Hertz. All ${ }^{1} \mathrm{H}$ and ${ }^{13} \mathrm{C}$ NMR assignments are corroborated by 2D experiments (HETCOR and COSY). Spectra available on request from denmark@scs.uiuc.edu.

Electron impact mass spectroscopy (EI) was performed at $70 \mathrm{eV}$ and chemical ionization (CI) spectra were obtained using methane as the carrier gas. Data are reported in the form of $\mathrm{m} / \mathrm{z}$ (intensity relative to base peak $=100)$. Infrared spectra $(I R)$ peaks are reported in $\mathrm{cm}^{-1}$ with indicated relative intensities: s (strong, 67-100\%); m (medium, 34-66\%); w (weak, 0-33\%). Analytical capillary gas chromatography (GC) was performed using a gas chromatograph fitted with a flame ionization detector $\left(\mathrm{H}_{2}\right.$ carrier gas, $\left.1 \mathrm{~mL} / \mathrm{min}\right)$. $\mathrm{GC}$ Method 1: Injections were made onto a Hewlett-Packard HP-5 50-m cross-linked 5\%-phenyl methyl silicone gum phase column. The detector temperature was $300{ }^{\circ} \mathrm{C}$. The column oven temperature program is as follows: 125 ${ }^{\circ} \mathrm{C}$ for 4 minutes, $125^{\circ} \mathrm{C}$ to $250^{\circ} \mathrm{C}$ ramp at $50^{\circ} \mathrm{C} /$ minute, $250{ }^{\circ} \mathrm{C}$ for 6 minutes. Total run time is 12.5 minutes. Retention times $\left(t_{R}\right)$ and integrated ratios were obtained from reporting integrators. Bulb-to-bulb distillations were performed on a Kugelrohr; boiling points (bp) corresponding to uncorrected air-bath temperatures (ABT). 


\section{Response Factors}

Response factors $\left(\mathrm{R}_{\mathrm{f}}\right)$ for quantitative GC analysis for GC Method 1 were obtained by the equation below:

Eq1: Response factor for $\mathrm{A}=($ area $\mathrm{A} *$ mmol biphenyl $) /(\mathrm{mmol} \mathrm{A} *$ area biphenyl $)$

\begin{tabular}{ccccc}
\hline mmol biphenyl & Area biphenyl & mmol 3a & Area 3a & Response factor \\
\hline 0.0492 & 27437 & 0.0395 & 11542 & 0.524 \\
0.0492 & 28707 & 0.0395 & 12971 & 0.563 \\
0.0492 & 17569 & 0.0395 & 8831 & 0.626 \\
0.0182 & 45830 & 0.0213 & 35817 & 0.668 \\
0.0182 & 43337 & 0.0213 & 40701 & 0.802 \\
0.0182 & 25151 & 0.0213 & 22347 & 0.759 \\
0.0253 & 24665 & 0.0326 & 27534 & 0.866 \\
0.0253 & 30122 & 0.0326 & 28639 & 0.738 \\
0.0253 & 35994 & 0.0326 & 35740 & 0.770 \\
\hline & & & Avg: & 0.701
\end{tabular}

\begin{tabular}{ccccc}
\hline mmol biphenyl & Area biphenyl & mmol 4-iodoanisole & Area 4-iodoanisole & Response factor \\
\hline 0.0389 & 20906 & 0.0406 & 7468 & 0.342 \\
0.0389 & 34354 & 0.0406 & 13373 & 0.373 \\
0.0389 & 22427 & 0.0406 & 7699 & 0.329 \\
0.0175 & 5928 & 0.0256 & 2957 & 0.341 \\
0.0175 & 9665 & 0.0256 & 5319 & 0.376 \\
0.0175 & 14950 & 0.0256 & 8801 & 0.402 \\
0.0454 & 51497 & 0.0607 & 26561 & 0.463 \\
0.0454 & 9996 & 0.0607 & 5594 & 0.502 \\
0.0454 & 49790 & 0.0607 & 28626 & 0.516 \\
\hline & & & Avg: & 0.386
\end{tabular}




\section{Preparation of Dimethyl-(1-heptynyl)silanol (2)}

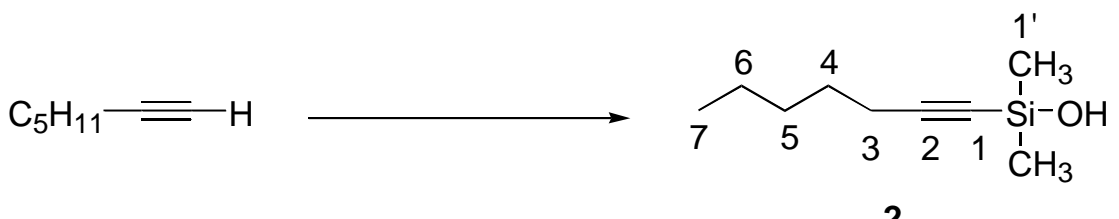

2

To an oven-dried, 250-mL round-bottom flask equipped with a magnetic stir bar was added 1-heptyne $(7.87 \mathrm{~mL}, 60.0 \mathrm{mmol})$ and $50 \mathrm{~mL}$ of anhydrous ether. The resulting solution was cooled to $-75{ }^{\circ} \mathrm{C}$. To the solution was slowly added $n$-butyllithium (1.55 $\mathrm{M}$ in hexane, 38.7 $\mathrm{mL}, 60.0 \mathrm{mmol}, 1.0$ equiv) and the solution stirred for $90 \mathrm{~min}$ at the same temperature. Chlorodimethylsilane (20.0 mL, $0.18 \mathrm{~mol}, 3.0$ equiv) was added and the solution was allowed to warm to room temperature over 2 hours. The reaction mixture was quenched with $10 \mathrm{~mL}$ of water then was extracted with water $(20 \mathrm{~mL})$. The aqueous phase was back-extracted with $2 \times 20$ $\mathrm{mL}$ of ether. The combined organics were washed with brine $(20 \mathrm{~mL})$ and then were dried over $\mathrm{MgSO}_{4}$. The volatile solvents were removed in vacuo to provide a yellow oil. The oil was taken up in $5 \mathrm{~mL}$ of hexanes and then was purified by silica gel chromatography $(60 \mathrm{~mm} \times 30 \mathrm{~cm})$ using hexanes to afford the intermediate silane, $(8.70 \mathrm{~g}, 94 \%)$ as an colorless oil.

To a 250-mL, round-bottom flask equipped with a magnetic stir bar was added the intermediate silane (11.40 g, $73.8 \mathrm{mmol}), 300 \mathrm{~mL}$ of acetonitrile, and water $(2.66 \mathrm{~mL}, 147.6$ mmol, 2.0 equiv). To the resulting solution was added $\left[\mathrm{RuCl}_{2}(p \text {-cymene })\right]_{2}(900 \mathrm{mg}, 1.47 \mathrm{mmol}$, 0.02 equiv). The solution was stirred at room temperature open to the atmosphere for $10 \mathrm{~min}$. The volatile solvents were then was removed in vacuo to provide a red oil. The oil was taken up in $20 \mathrm{~mL}$ of hexanes and then was purified by silica gel chromatography $(30 \mathrm{~mm} \times 30 \mathrm{~cm}) \mathrm{using}$ 
hexanes/ethyl acetate, $5 / 1$ to produce a colorless oil. Short-path distillation $\left(100{ }^{\circ} \mathrm{C}, 1.0 \mathrm{mmHg}\right)$ afforded $10.58 \mathrm{~g}(84 \%)$ of dimethyl(1-heptynyl)silanol as a colorless oil.

Analytical Data for 2

Data for Dimethyl(1-heptynyl)silanol (2):

bp: $\quad 100{ }^{0} \mathrm{C}(1.0 \mathrm{mmHg}, \mathrm{ABT})$

${ }^{1} \underline{\mathrm{H} \mathrm{NMR}}: \quad\left(500 \mathrm{MHz}, \mathrm{CHCl}_{3}\right)$

$2.22\left(\mathrm{t}, J=7.5,2 \mathrm{H}, \mathrm{H}_{2} \mathrm{C}(3)\right), 2.04(\mathrm{~s}, 1 \mathrm{H},-\mathrm{OH}), 1.52$ (pent, $\left.J=7.5,2 \mathrm{H}, \mathrm{H}_{2} \mathrm{C}(4)\right)$, 1.30-1.36 (m, $4 \mathrm{H}, \mathrm{H}_{2} \mathrm{C}\left(5\right.$ and 6)), $0.90\left(\mathrm{t}, J=7,3 \mathrm{H}, \mathrm{H}_{3} \mathrm{C}(7)\right), 0.27$ (s, $6 \mathrm{H}$, $\left.\mathrm{H}_{3} \mathrm{C}\left(1^{\prime}\right)\right)$

${ }^{13} \mathrm{C} \mathrm{NMR:} \quad\left(126 \mathrm{MHz}, \mathrm{CHCl}_{3}\right)$

$107.87(\mathrm{C}(1)), 83.34(\mathrm{C}(2)), 30.97(\mathrm{C}(5)), 28.06(\mathrm{C}(4)), 22.11(\mathrm{C}(6)), 19.62$ $(\mathrm{C}(3)), 13.90(\mathrm{C}(7)), 1.61\left(\mathrm{C}\left(1^{\prime}\right)\right)$

IR: (neat)

3291 (s), 2960 (s), 2935 (s), 2862 (s), 2176 (s), 1467 (m), 1429 (m), 1253 (s), 1049 (m), 900, (s), 830, (s), 787 (s)

$\underline{\mathrm{MS}}: \quad(\mathrm{EI}, 70 \mathrm{ev})$

155 ( M-15, 100), 98 (12), 85 (11), 75 (54), 61 (15)

TLC: $\quad R_{f} 0.07$ (hexane/EtOAc, 19/1) [silica gel, $\mathrm{KMnO}_{4}$ ]

Analysis: $\quad \mathrm{C}_{17} \mathrm{H}_{18}(222.32)$

Calcd: $\quad$ C, 63.47; H, 10.65\%

Found: $\quad$ C, 63.21; H, $10.85 \%$ 
General Procedure I: Competition Study of Palladium-Catalyzed Cross-Coupling of Alkynylsilicon Compounds with 4-Iodoanisole

To an oven-dried, 5-mL, round-bottom flask equipped with a magnetic stir bar was added $128.2 \mathrm{mg}$ (1.0 mmol, 2.0 equiv) of potassium trimethylsilanolate under a dry argon atmosphere. To this was added sequentially copper(I) iodide ( $4.8 \mathrm{mg}, 0.025 \mathrm{mmol}, 0.05$ equiv), biphenyl (49.9 mg), $1 \mathrm{~mL}$ of DME, dimethyl(1-heptynyl)silanol (93.7 mg, $0.55 \mathrm{mmol}, 1.1$ equiv), 4-iodoanisole (117.0 mg, $1.0 \mathrm{mmol}, 1.0$ equiv), and $\mathrm{PdCl}_{2}\left(\mathrm{PPh}_{3}\right)_{2}(8.6 \mathrm{mg}, 0.0125 \mathrm{mmol}, 0.025$ equiv). The progress of the reaction was monitored by GC as follows: an aliquot of the reaction mixture was filtered through a small amount of silica gel with EtOAc and was analyzed by GC method 1 .

\section{Table 1, entry 1}

Following General Procedure I, potassium trimethylsilanolate (128.2 mg, $1.0 \mathrm{mmol}, 2.0$ equiv.), biphenyl (50.5 mg), $1 \mathrm{~mL}$ DME, 1-heptyne (52.9 mg, $0.55 \mathrm{mmol}, 1.1$ equiv), 4-iodoanisole (117.0 mg, $1.0 \mathrm{mmol}, 1.0$ equiv), and $\mathrm{PdCl}_{2}\left(\mathrm{PPh}_{3}\right)_{2}(8.6 \mathrm{mg}, 0.0125 \mathrm{mmol}, 0.025$ equiv) were combined and stirred at $\mathrm{rt}$ for $3 \mathrm{~h}$. An aliquot of the mixture was analyzed by GC Method 1: 4-iodoanisole, $t_{\mathrm{R}} 7.28 \min (8608,90 \%), \mathbf{3 a}, t_{\mathrm{R}} 9.78 \min (947,2 \%)$.

\section{Table 1, entry 2}

Following General Procedure I, potassium trimethylsilanolate $(128.2 \mathrm{mg}, 1.0 \mathrm{mmol}, 2.0$ equiv.), biphenyl (50.5 mg), $1 \mathrm{~mL}$ DME, 1-trimethylsilyl-1-heptyne (92.6 mg, $0.55 \mathrm{mmol}, 1.1$ equiv), 4-iodoanisole (117.0 mg, $1.0 \mathrm{mmol}, 1.0$ equiv), and $\mathrm{PdCl}_{2}\left(\mathrm{PPh}_{3}\right)_{2}(8.6 \mathrm{mg}, 0.0125 \mathrm{mmol}$, 
0.025 equiv) were combined and stirred at $\mathrm{rt}$ for $3 \mathrm{~h}$. An aliquot of the mixture was analyzed by GC Method 1: 4-iodoanisole, $t_{\mathrm{R}} 7.28 \min (4533,106 \%), 3 \mathbf{a}, t_{\mathrm{R}} 9.78 \min (0,0 \%)$.

\section{Table 1, entry 3}

Following General Procedure I, potassium trimethylsilanolate (128.2 mg, $1.0 \mathrm{mmol}, 2.0$ equiv.), biphenyl (48.8 mg), $1 \mathrm{~mL}$ DME, dimethyl(1-heptynyl)silanol (93.7 mg, $0.55 \mathrm{mmol}, 1.1$ equiv), 4-iodoanisole (117.0 mg, $1.0 \mathrm{mmol}, 1.0$ equiv), and $\mathrm{PdCl}_{2}\left(\mathrm{PPh}_{3}\right)_{2}(8.6 \mathrm{mg}, 0.0125 \mathrm{mmol}$, 0.025 equiv) were combined and stirred at $\mathrm{rt}$ for $3 \mathrm{~h}$. An aliquot of the mixture was analyzed by GC Method 1: 4-iodoanisole, $t_{\mathrm{R}} 7.28 \min (1687,24 \%), \mathbf{3 a}, t_{\mathrm{R}} 9.78 \min (3131,25 \%)$.

\section{Table 1, entry 4}

Following General Procedure I, potassium trimethylsilanolate (128.2 mg, $1.0 \mathrm{mmol}, 2.0$ equiv.), copper(I) iodide (4.8 mg, $0.025 \mathrm{mmol}, 0.05$ equiv), biphenyl (50.4 mg), $1 \mathrm{~mL} \mathrm{DME}$, 1-heptyne (52.9 mg, $0.55 \mathrm{mmol}, 1.1$ equiv), 4-iodoanisole (117.0 mg, $1.0 \mathrm{mmol}, 1.0$ equiv), and $\mathrm{PdCl}_{2}\left(\mathrm{PPh}_{3}\right)_{2}(8.6 \mathrm{mg}, 0.0125 \mathrm{mmol}, 0.025$ equiv $)$ were combined and stirred at $\mathrm{rt}$ for $3 \mathrm{~h}$. An aliquot of the mixture was analyzed by GC Method 1: 4-iodoanisole, $t_{\mathrm{R}} 7.28 \min (14175,118 \%)$, 3a, $t_{\mathrm{R}} 9.78 \min (1051,14 \%)$.

\section{Table 1, entry 5}

Following General Procedure I, potassium trimethylsilanolate (128.2 mg, $1.0 \mathrm{mmol}, 2.0$ equiv.), copper(I) iodide (4.8 mg, $0.025 \mathrm{mmol}, 0.05$ equiv), biphenyl (47.0 mg), $1 \mathrm{~mL} \mathrm{DME}$, 1-trimethylsilyl-1-heptyne $(92.6 \mathrm{mg}, 0.55 \mathrm{mmol}, 1.1$ equiv), 4-iodoanisole $(117.0 \mathrm{mg}, 1.0 \mathrm{mmol}$, 1.0 equiv), and $\mathrm{PdCl}_{2}\left(\mathrm{PPh}_{3}\right)_{2}(8.6 \mathrm{mg}, 0.0125 \mathrm{mmol}, 0.025$ equiv $)$ were combined and stirred at $\mathrm{rt}$ 
for $3 \mathrm{~h}$. An aliquot of the mixture was analyzed by GC Method 1: 4-iodoanisole, $t_{\mathrm{R}} 7.28 \mathrm{~min}$ (14908, 113\%), 3a, $t_{\mathrm{R}} 9.78 \min (1519,16 \%)$.

\section{Table 1, entry 6}

Following General Procedure I, potassium trimethylsilanolate (128.2 mg, $1.0 \mathrm{mmol}, 2.0$ equiv.), (I) iodide (4.8 mg, $0.025 \mathrm{mmol}, 0.05$ equiv), biphenyl (49.9 mg), $1 \mathrm{~mL} \mathrm{DME}$, dimethyl(1-heptynyl)silanol (93.7 mg, $0.55 \mathrm{mmol}, 1.1$ equiv), 4-iodoanisole (117.0 mg, 1.0 mmol, 1.0 equiv), and $\mathrm{PdCl}_{2}\left(\mathrm{PPh}_{3}\right)_{2}(8.6 \mathrm{mg}, 0.0125 \mathrm{mmol}, 0.025$ equiv) were combined and stirred at $\mathrm{rt}$ for $3 \mathrm{~h}$. An aliquot of the mixture was analyzed by GC Method 1: 4-iodoanisole, $t_{\mathrm{R}}$ $7.28 \min (0,0 \%), \mathbf{3 a}, t_{\mathrm{R}} 9.78 \min (46517,99 \%)$.

\section{Table 1, entry 7}

Following General Procedure I, tetrabutylammonium fluoride (315 mg, $1.0 \mathrm{mmol}, 2.0$ equiv.), biphenyl (49.2 mg), $1 \mathrm{~mL}$ DME, 1-heptyne (52.9 mg, $0.55 \mathrm{mmol}, 1.1$ equiv), 4-iodoanisole (117.0 mg, $1.0 \mathrm{mmol}, 1.0$ equiv), and $\mathrm{PdCl}_{2}\left(\mathrm{PPh}_{3}\right)_{2}(8.6 \mathrm{mg}, 0.0125 \mathrm{mmol}, 0.025$ equiv) were combined and stirred at $\mathrm{rt}$ for $3 \mathrm{~h}$. An aliquot of the mixture was analyzed by GC Method 1: 4-iodoanisole, $t_{\mathrm{R}} 7.28 \min (3105,82 \%), \mathbf{3 a}, t_{\mathrm{R}} 9.78 \min (1196,17 \%)$.

\section{Table 1, entry 8}

Following General Procedure I, tetrabutylammonium fluoride (315 mg, $1.0 \mathrm{mmol}, 2.0$ equiv.), biphenyl (49.6 mg), $1 \mathrm{~mL}$ DME, 1-trimethylsilyl-1-heptyne (92.6 mg, $0.55 \mathrm{mmol}, 1.1$ equiv), 4-iodoanisole (117.0 mg, $1.0 \mathrm{mmol}, 1.0$ equiv), and $\mathrm{PdCl}_{2}\left(\mathrm{PPh}_{3}\right)_{2}(8.6 \mathrm{mg}, 0.0125 \mathrm{mmol}$, 
0.025 equiv) were combined and stirred at $\mathrm{rt}$ for $3 \mathrm{~h}$. An aliquot of the mixture was analyzed by GC Method 1: 4-iodoanisole, $t_{\mathrm{R}} 7.28 \min (7212,83 \%), \mathbf{3 a}, t_{\mathrm{R}} 9.78 \min (3526,22 \%)$.

\section{Table 1, entry 9}

Following General Procedure I, tetrabutylammonium fluoride (315 mg, $1.0 \mathrm{mmol}, 2.0$ equiv.), biphenyl (51.3 mg), $1 \mathrm{~mL}$ DME, dimethyl(1-heptynyl)silanol (93.7 mg, $0.55 \mathrm{mmol}, 1.1$ equiv), 4-iodoanisole (117.0 mg, $1.0 \mathrm{mmol}, 1.0$ equiv), and $\mathrm{PdCl}_{2}\left(\mathrm{PPh}_{3}\right)_{2}(8.6 \mathrm{mg}, 0.0125 \mathrm{mmol}$, 0.025 equiv) were combined and stirred at $\mathrm{rt}$ for $3 \mathrm{~h}$. An aliquot of the mixture was analyzed by GC Method 1: 4-iodoanisole, $t_{\mathrm{R}} 7.28 \min (4218,42 \%), 3 \mathbf{a}, t_{\mathrm{R}} 9.78 \min (8459,47 \%)$. 
General Procedure II: Cross-Coupling of Dimethyl(1-heptynyl)silanol (2) with Aryl Iodides. Preparation of 1-(1-Heptynyl)-4-methoxybenzene (3a) ${ }^{1}$

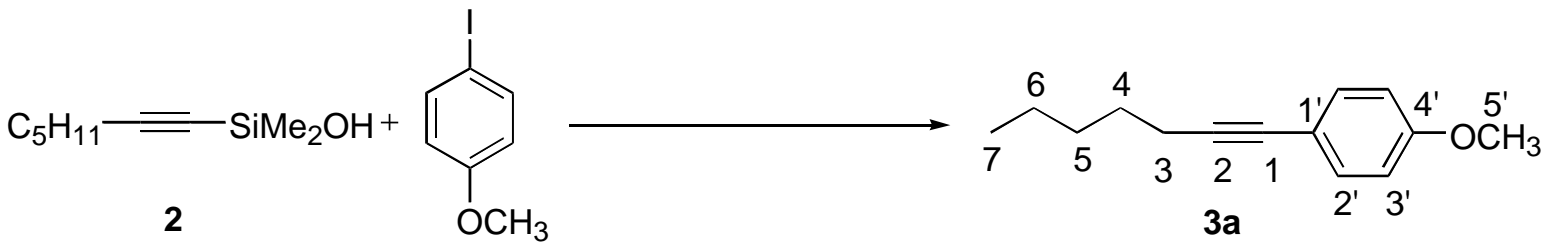

To an oven-dried, 10-mL, round-bottom flask equipped with a magnetic stir bar was added $256.0 \mathrm{mg}$ ( $2.0 \mathrm{mmol}, 2.0$ equiv) of potassium trimethylsilanolate under a dry argon atmosphere. To this was added sequentially copper(I) iodide ( $9.6 \mathrm{mg}, 0.05 \mathrm{mmol}, 0.05$ equiv), 2 $\mathrm{mL}$ of DME, dimethyl(1-heptynyl)silanol (187.0 mg, $1.1 \mathrm{mmol}, 1.1$ equiv), 4-iodoanisole (234.0 mg, $1.0 \mathrm{mmol}, 1.0$ equiv), and $\mathrm{PdCl}_{2}\left(\mathrm{PPh}_{3}\right)_{2}$ (17.2 mg, $0.025 \mathrm{mmol}, 0.025$ equiv). The resulting solution was stirred at room temperature for $3 \mathrm{~h}$ under a dry argon atmosphere. The reaction mixture was then filtered through a silica plug $(3 \mathrm{~g})$ with $75 \mathrm{~mL}$ of anhydrous $\mathrm{Et}_{2} \mathrm{O}$. The volatile solvents were removed in vacuo to afford a yellow oil. The oil was taken up in $3 \mathrm{~mL}$ of ether and then was purified by silica gel column chromatography $(30 \mathrm{~mm}$ x $30 \mathrm{~cm}$ ) using pentane/ether, 19/1 to give a pale yellow oil. Bulb-to-bulb distillation (ABT $150{ }^{\circ} \mathrm{C}, 1.0 \mathrm{mmHg}$ ) afforded $191 \mathrm{mg}(95 \%)$ of $\mathbf{3 a}$ as a colorless oil.

Data for 1-(1-Heptynyl)-4-methoxy-benzene (3a):

bp: $\quad 150{ }^{\circ} \mathrm{C}(1.0 \mathrm{mmHg}, \mathrm{ABT})$

1ㅅN $\underline{\mathrm{HMR}}: \quad\left(500 \mathrm{MHz}, \mathrm{CHCl}_{3}\right)$

$7.26\left(\mathrm{dd}, J=2,7,2 \mathrm{H}, 2 \times \mathrm{HC}\left(3^{\prime}\right)\right), 6.75\left(\mathrm{dd}, J=2,7,2 \mathrm{H}, 2 \times \mathrm{HC}\left(2^{\prime}\right)\right), 3.74$ (s, 3 $\left.\mathrm{H}, \mathrm{H}_{3} \mathrm{C}\left(5^{\prime}\right)\right), 2.32$ (t, $\left.J=7.5,2 \mathrm{H}, \mathrm{H}_{2} \mathrm{C}(3)\right), 1.52$ (pent, $\left.J=7.5,2 \mathrm{H}, \mathrm{H}_{2} \mathrm{C}(4)\right), 1.27-$ $1.35\left(\mathrm{~m}, 4 \mathrm{H}, \mathrm{H}_{2} \mathrm{C}\left(5\right.\right.$ and 6), $0.86\left(\mathrm{t}, J=7.5,3 \mathrm{H}, \mathrm{H}_{3} \mathrm{C}(7)\right)$ 
${ }^{13}$ C NMR: $\quad\left(126 \mathrm{MHz}, \mathrm{CHCl}_{3}\right)$

159.21 (C(4’)), $133.10\left(\mathrm{C}\left(2^{\prime}\right)\right), 116.49$ (C(3’)), $114.03\left(\mathrm{C}\left(1^{\prime}\right)\right), 89.07(\mathrm{C}(1))$, $80.42(\mathrm{C}(2)), 55.48\left(\mathrm{C}\left(5^{\prime}\right)\right), 31.36(\mathrm{C}(5)), 28.80(\mathrm{C}(4)), 22.47(\mathrm{C}(6)), 19.60(\mathrm{C}(3))$, $14.24(\mathrm{C}(7))$

GC: $\quad t_{\mathrm{R}} 9.35 \mathrm{~min}\left(\mathrm{HP}-5,15 \mathrm{psi}, 175{ }^{\circ} \mathrm{C}\right.$ for $7 \mathrm{~min}, 25{ }^{\circ} \mathrm{C} / \mathrm{min}$ to $\left.225^{\circ} \mathrm{C}\right)$

Preparation of 1-Phenyl-1-heptyne (3b) (Table 2, entry 2) ${ }^{2}$

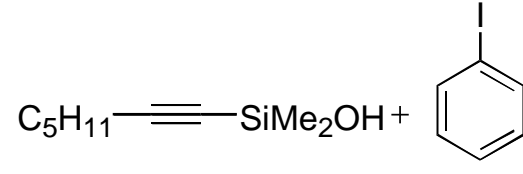

2

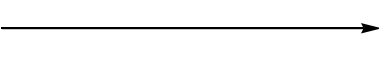

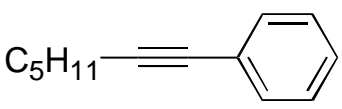

$3 b$

Following General Procedure II, iodobenzene (408 mg, $2.00 \mathrm{mmol})$ was combined with potassium trimethylsilanolate (512 $\mathrm{mg}, 4.00 \mathrm{mmol}, 2.0$ equiv), copper(I) iodide (19 $\mathrm{mg}, 0.10$ mmol, 0.05 equiv), $4 \mathrm{~mL}$ of DME, dimethyl(1-heptynyl)silanol (375 mg, $2.2 \mathrm{mmol}, 1.1 \mathrm{equiv})$, and $\mathrm{PdCl}_{2}\left(\mathrm{PPh}_{3}\right)_{2}(35 \mathrm{mg}, 0.05 \mathrm{mmol}, 0.025$ equiv). The product $3 \mathbf{b}$ was obtained as a colorless oil in $93 \%$ yield $(320 \mathrm{mg})$.

Analytical data for 1-Phenyl-1-heptyne (3b)

${ }^{1}$ H NMR: $\quad\left(500 \mathrm{MHz}, \mathrm{CHCl}_{3}\right)$

7.39-7.40 (m, $2 \mathrm{H}), 7.25-7.38(\mathrm{~m}, 3 \mathrm{H}), 2.40\left(\mathrm{t}, J=7.0,2 \mathrm{H}, \mathrm{H}_{2} \mathrm{C}(3)\right), 1.61$ (pent, $J$ $\left.=7.5,2 \mathrm{H}, \mathrm{H}_{2} \mathrm{C}(4)\right), 1.33-1.42\left(\mathrm{~m}, 4 \mathrm{H}, \mathrm{H}_{2} \mathrm{C}(5\right.$ and 6$), 0.92(\mathrm{t}, J=7.03 \mathrm{H}$, $\left.\mathrm{H}_{3} \mathrm{C}(7)\right)$ 


\section{Preparation of 1-(1-Heptynyl)naphthalene (3c) (Table 2, entry 3)}
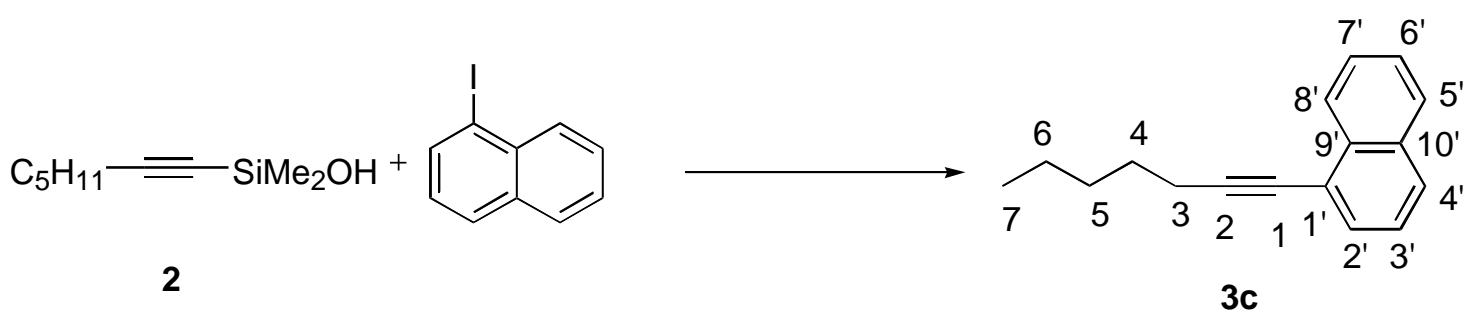

Following General Procedure II, 1-iodonaphthalene (508 mg, $2.00 \mathrm{mmol}$ ) was combined with potassium trimethylsilanolate $(512 \mathrm{mg}, 4.00 \mathrm{mmol}, 2.0$ equiv), copper(I) iodide (19 mg, $0.10 \mathrm{mmol}, 0.05$ equiv), $4 \mathrm{~mL}$ of DME, dimethyl(1-heptynyl)silanol (375 mg, $2.2 \mathrm{mmol}, 1.1$ equiv), and $\mathrm{PdCl}_{2}\left(\mathrm{PPh}_{3}\right)_{2}$ (35 mg, $0.05 \mathrm{mmol}, 0.025$ equiv). The product $3 \mathrm{c}$ was obtained as a colorless oil in $87 \%$ yield $(385 \mathrm{mg})$.

Data for 1-(1-Heptynyl)naphthalene (3c):

bp: $\quad 160{ }^{\circ} \mathrm{C}(0.5 \mathrm{mmHg}, \mathrm{ABT})$

${ }^{1} \underline{\mathrm{H} \text { NMR: }}: \quad\left(500 \mathrm{MHz}, \mathrm{CHCl}_{3}\right)$

$8.37\left(\mathrm{~d}, J=8.5,1 \mathrm{H}, \mathrm{HC}\left(8^{\prime}\right)\right), 7.84\left(\mathrm{~d}, J=8.5,1 \mathrm{H}, \mathrm{HC}\left(2^{\prime}\right)\right), 7.79(\mathrm{~d}, J=8.5,1$ H, $\left.\mathrm{HC}\left(5^{\prime}\right)\right), 7.64\left(\mathrm{~d}, J=7.5,1 \mathrm{H}, \mathrm{HC}\left(4^{\prime}\right)\right), 7.57\left(\mathrm{t}, J=7.5,1 \mathrm{H}, \mathrm{HC}\left(7^{\prime}\right)\right), 7.51(\mathrm{t}$, $\left.J=7.5,1 \mathrm{H}, \mathrm{HC}\left(6^{\prime}\right)\right), 7.41\left(\mathrm{t}, J=7.5,1 \mathrm{H}, \mathrm{HC}\left(3^{\prime}\right)\right), 2.59(\mathrm{t}, J=7.0,2 \mathrm{H}$, $\mathrm{H}_{2} \mathrm{C}(3)$ ), 1.74 (pent, $\left.J=7.5,2 \mathrm{H}, \mathrm{H}_{2} \mathrm{C}(4)\right), 1.54$ (pent, $J=7.5,2 \mathrm{H}, \mathrm{H}_{2} \mathrm{C}(5)$ ), 1.42 (pent, $\left.J=7.5,2 \mathrm{H}, \mathrm{H}_{2} \mathrm{C}(6)\right), 0.97\left(\mathrm{t}, J=7.0,3 \mathrm{H}, \mathrm{H}_{3} \mathrm{C}(7)\right.$ )

${ }^{13} \mathrm{C} \mathrm{NMR}: \quad\left(126 \mathrm{MHz}, \mathrm{CHCl}_{3}\right)$

$133.5\left(\mathrm{C}\left(9^{\prime}\right)\right), 133.2\left(\mathrm{C}\left(10^{\prime}\right)\right), 130.0\left(\mathrm{C}\left(2^{\prime}\right)\right), 128.2\left(\mathrm{C}\left(4^{\prime}\right)\right), 127.8\left(\mathrm{C}\left(5^{\prime}\right)\right), 126.4$ (C(6’)), $126.3\left(\mathrm{C}\left(8^{\prime}\right)\right), 126.2\left(\mathrm{C}\left(7^{\prime}\right)\right), 125.2\left(\mathrm{C}\left(3^{\prime}\right)\right), 121.8\left(\mathrm{C}\left(1^{\prime}\right)\right), 95.6(\mathrm{C}(1))$, $78.5(\mathrm{C}(2)), 31.2(\mathrm{C}(5)), 28.6(\mathrm{C}(4)), 22.4(\mathrm{C}(6)), 19.7(\mathrm{C}(3)), 14.0(\mathrm{C}(7))$ 
IR: $\quad$ (neat)

3058 (s), 2931 (s), 2859 (s), 2222 (m), 1928 (w,b), 1586 (s), 1463 (s), 1395 (s), $1328(\mathrm{~m}), 1016(\mathrm{~m}), 798(\mathrm{~s}), 773(\mathrm{~s})$

MS: $\quad(E I, 70 \mathrm{ev})$

$222\left(\mathrm{M}^{+}, 38\right), 193$ (25), 179 (30), 178 (24), 167 (34), 166 (19), 165 (100), 164 (20), 163 (26), $152(22), 139$ (12)

TLC: $\quad R_{f} 0.52$ (hexane/EtOAc, 19/1) [silica gel, $\mathrm{KMnO}_{4}$ ]

GC: $\quad t \mathrm{R} 12.40 \mathrm{~min}\left(\mathrm{HP}-5,15 \mathrm{psi}, 175{ }^{\circ} \mathrm{C}\right.$ for $7 \mathrm{~min}, 25{ }^{\circ} \mathrm{C} / \mathrm{min}$ to $\left.225{ }^{\circ} \mathrm{C}\right)$

Analysis: $\quad \mathrm{C}_{17} \mathrm{H}_{18}(222.32)$

Calcd: $\quad$ C, $91.84 ; \quad H, 8.16 \%$

Found: $\quad$ C, $91.83 ; \quad H, 8.21 \%$

\section{Preparation of 4-(1-Heptynyl)benzonitrile (3d) (Table 2, entry 4)}

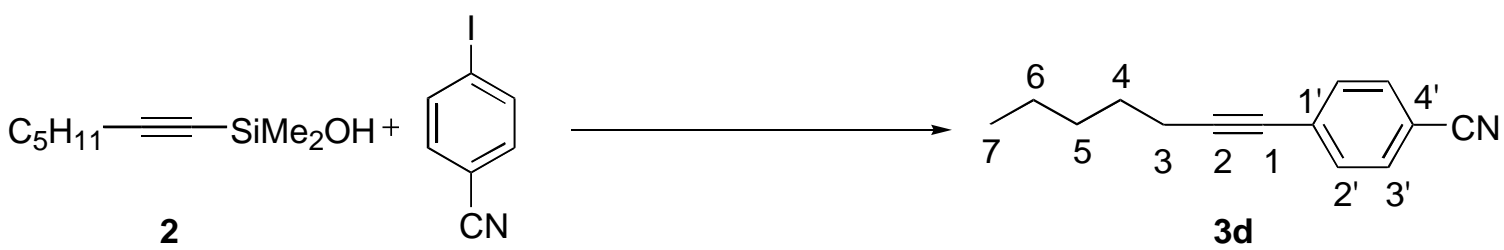

Following General Procedure II, 4-iodobenzonitrile (229 mg, $1.00 \mathrm{mmol}$ ) was combined with potassium trimethylsilanolate $(256 \mathrm{mg}, 2.00 \mathrm{mmol}, 2.0$ equiv), copper(I) iodide (9.6 mg, $0.05 \mathrm{mmol}, 0.05$ equiv), $2 \mathrm{~mL}$ of DME, dimethyl(1-heptynyl)silanol (187 mg, $1.1 \mathrm{mmol}, 1.1$ equiv), and $\mathrm{PdCl}_{2}\left(\mathrm{PPh}_{3}\right)_{2}(17.2 \mathrm{mg}, 0.025 \mathrm{mmol}, 0.025$ equiv). The product $\mathbf{3 d}$ was obtained as a colorless oil in $83 \%$ yield $(176 \mathrm{mg})$. 
Data for 4-(1-Heptynyl)benzonitrile (3d):

bp: $\quad 150{ }^{\circ} \mathrm{C}(0.5 \mathrm{mmHg}, \mathrm{ABT})$

${ }^{1}$ H NMR: $\quad\left(500 \mathrm{MHz}, \mathrm{CHCl}_{3}\right)$

$7.56\left(\mathrm{dd}, J=2,6.5,2 \mathrm{H}, 2 \times \mathrm{HC}\left(3^{\prime}\right)\right), 6.75\left(\mathrm{dd}, J=2,7,2 \mathrm{H}, 2 \times \mathrm{HC}\left(2^{\prime}\right)\right), 2.42(\mathrm{t}$, $\left.J=7.5,2 \mathrm{H}, \mathrm{H}_{2} \mathrm{C}(3)\right), 1.61$ (pent, $\left.J=7.0,2 \mathrm{H}, \mathrm{H}_{2} \mathrm{C}(4)\right), 1.25-1.44$ (m, $4 \mathrm{H}, \mathrm{H}_{2} \mathrm{C}(5$ and 6), $0.93\left(\mathrm{t}, J=7.0,3 \mathrm{H}, \mathrm{H}_{3} \mathrm{C}(7)\right)$

${ }^{13}$ C NMR: $\quad\left(126 \mathrm{MHz}, \mathrm{CHCl}_{3}\right)$

$132.3\left(\mathrm{C}\left(3^{\prime}\right)\right), 132.1\left(\mathrm{C}\left(2^{\prime}\right)\right), 129.4\left(\mathrm{C}\left(1^{\prime}\right)\right), 118.8(\mathrm{CN}), 111.0\left(\mathrm{C}\left(4^{\prime}\right)\right), 95.9$ (C(1)), $79.6(\mathrm{C}(2)), 31.3(\mathrm{C}(5)), 28.3(\mathrm{C}(4)), 22.4(\mathrm{C}(6)), 19.7(\mathrm{C}(3)), 14.1(\mathrm{C}(7))$

IR: (neat)

2932 (s), 2860 (s), 2227 (s), 1921 (w,b), 1604 (s), 1500 (s), 1466 (s), 1428 (m), $1405(\mathrm{~m}), 1330(\mathrm{~m}), 1176(\mathrm{~s}), 839(\mathrm{~s})$

MS: $\quad(E I, 70 \mathrm{ev})$

197 ( $\left.\mathrm{M}^{+}, 32\right)$, 182, (16), 169 (19), 168 (100), 167 (12), 156 (12), 155 (39), 154

(54), 153 (28), 143 (15), 142 (43), 141 (41), 140 (95), 128 (18), 127 (44), 116 (20), 115 (21), 114 (21), 113 (27), 81 (22), 76 (10), 63 (20) 56 (24)

TLC: $\quad R_{f} 0.31$ (hexane/EtOAc, 19/1) [silica gel, $\mathrm{KMnO}_{4}$ ]

GC: $\quad t_{\mathrm{R}} 12.40 \mathrm{~min}\left(\mathrm{HP}-5,15 \mathrm{psi}, 175^{\circ} \mathrm{C}\right.$ for $7 \mathrm{~min}, 25{ }^{\circ} \mathrm{C} / \min$ to $\left.225^{\circ} \mathrm{C}\right)$

Analysis: $\quad \mathrm{C}_{14} \mathrm{H}_{15} \mathrm{~N}(197.28)$

Calcd: $\quad$ C, 85.24; H, $7.66 \quad$ N, 7.10\%

Found: $\quad$ C, 85.12; $\quad \mathrm{H}, 7.72 \quad \mathrm{~N}, 7.18 \%$ 
Preparation of 1-(1-Heptynyl)-4-methylbenzene (3e) (Table 2, entry 5)

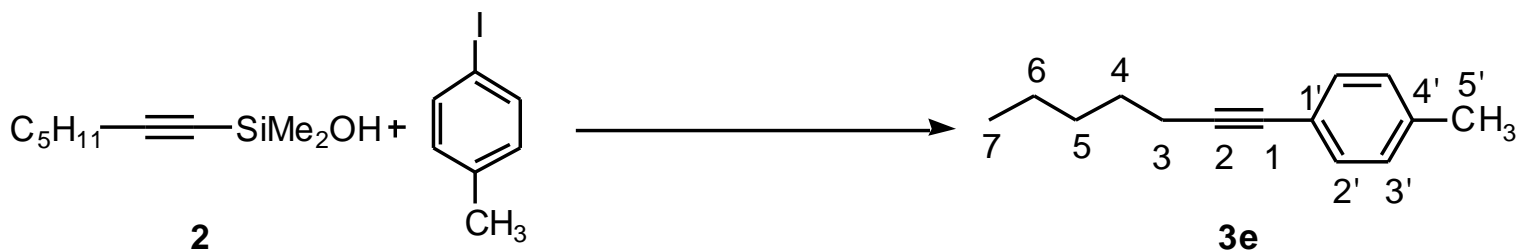

Following General Procedure II, 4-iodotoluene (218 mg, $1.00 \mathrm{mmol})$ was combined with potassium trimethylsilanolate (256 mg, $2.00 \mathrm{mmol}, 2.0$ equiv), copper(I) iodide (9.6 mg, 0.05 mmol, 0.05 equiv), $2 \mathrm{~mL}$ of DME, dimethyl(1-heptynyl)silanol (187 mg, $1.1 \mathrm{mmol}, 1.1 \mathrm{equiv})$, and $\mathrm{PdCl}_{2}\left(\mathrm{PPh}_{3}\right)_{2}(17.2 \mathrm{mg}, 0.025 \mathrm{mmol}, 0.025$ equiv $)$. The product 3e was obtained as a colorless oil in $91 \%$ yield (169 $\mathrm{mg})$.

Data for 1-(1-Heptynyl)-4-methylbenzene (3e):

bp: $\quad 150{ }^{0} \mathrm{C}(0.5 \mathrm{mmHg}, \mathrm{ABT})$

${ }^{1}$ H NMR: $\quad\left(500 \mathrm{MHz}, \mathrm{CHCl}_{3}\right)$

$7.29\left(\mathrm{~d}, J=8.0,2 \mathrm{H}, 2 \times \mathrm{HC}\left(2^{\prime}\right)\right), 7.08\left(\mathrm{~d}, J=7.5,2 \mathrm{H}, 2 \times \mathrm{HC}\left(3^{\prime}\right)\right), 2.39$ (t, $J$ $\left.=7.0,2 \mathrm{H}, \mathrm{H}_{2} \mathrm{C}(3)\right), 2.33\left(\mathrm{~s}, 3 \mathrm{H}, \mathrm{H}_{3} \mathrm{C}\left(5^{\prime}\right)\right), 1.61$ (pent, $\left.J=7.5,2 \mathrm{H}, \mathrm{H}_{2} \mathrm{C}(4)\right), 1.34-$ $1.46\left(\mathrm{~m}, 4 \mathrm{H}, \mathrm{H}_{2} \mathrm{C}\left(5\right.\right.$ and 6), $0.93\left(\mathrm{t}, J=7.5,3 \mathrm{H}, \mathrm{H}_{3} \mathrm{C}(7)\right)$

${ }^{13} \underline{\mathrm{C} \mathrm{NMR}}: \quad\left(126 \mathrm{MHz}, \mathrm{CHCl}_{3}\right)$

137.1 (C(4')), 131.1 (C(2')), 128.7 (C(3’)), 120.7 (C(1')), 89.4 (C(1)), 80.3 (C(2)), $\left.30.8(\mathrm{C}(5)), 28.2(\mathrm{C}(4)), 21.9(\mathrm{C}(6)), 21.1 \mathrm{C}\left(5^{\prime}\right)\right), 19.1(\mathrm{C}(3)), 13.7(\mathrm{C}(7))$ IR: $\quad$ (neat) 2957 (s), 2932 (s), 2860 (s), 2200 (w), $1701(w), 1509$ (s), $1466(\mathrm{~s}), 1106(\mathrm{w})$ 
MS: $\quad(E I, 70 \mathrm{ev})$

$186\left(\mathrm{M}^{+}, 63\right), 171$ (12), 158 (12), 157 (54), 145 (12), 144 (18), 143 (41), 142

(29), 141 (15), 131 (66), 130 (15), 129 (100), 128 (42), 127 (16), 116 (16), 115

(30), 105 (16), 91 (15), 77 (12)

TLC: $\quad R_{f} 0.55$ (hexane/EtOAc, 19/1) [silica gel, $\mathrm{KMnO}_{4}$ ]

GC: $\quad t_{\mathrm{R}} 10.00 \min \left(\mathrm{HP}-5,15 \mathrm{psi}, 125{ }^{\circ} \mathrm{C}\right.$ for $4 \mathrm{~min}, 50{ }^{\circ} \mathrm{C} / \mathrm{min}$ to $\left.250{ }^{\circ} \mathrm{C}\right)$

Analysis: $\quad \mathrm{C}_{14} \mathrm{H}_{18}(186.29)$

Calcd: $\quad$ C, $90.26 ; \quad$ H, $9.74 \%$

Found: $\quad$ C, $90.33 ; \quad H, 9.82 \%$

Preparation of 1-(1-Heptynyl)-4-nitrobenzene (3f) (Table 2, entry 6)

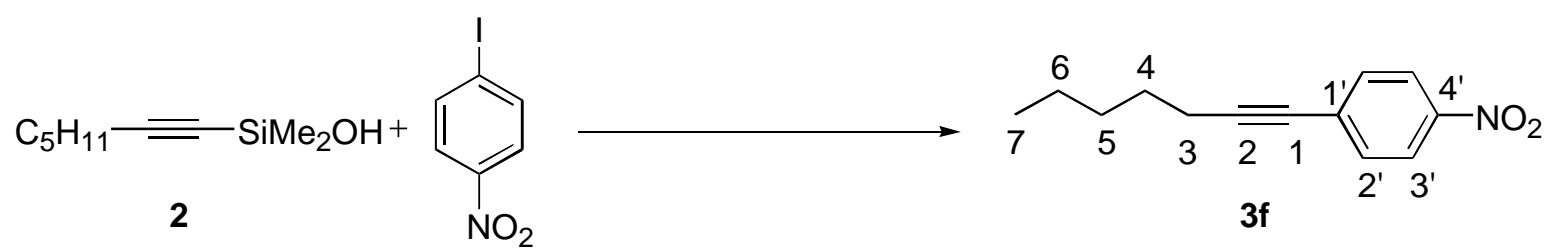

Following General Procedure II, 4-iodonitrobenzene (498 mg, 2.00 mmol) was combined with potassium trimethylsilanolate $(512 \mathrm{mg}, 4.00 \mathrm{mmol}, 2.0$ equiv), copper(I) iodide (19 mg, $0.10 \mathrm{mmol}, 0.05$ equiv), $4 \mathrm{~mL}$ of DME, dimethyl(1-heptynyl)silanol (375 mg, $2.2 \mathrm{mmol}, 1.1$ equiv), and $\mathrm{PdCl}_{2}\left(\mathrm{PPh}_{3}\right)_{2}(35 \mathrm{mg}, 0.05 \mathrm{mmol}, 0.025$ equiv). The product $\mathbf{3 f}$ was obtained as a bright yellow oil in $75 \%$ yield $(328 \mathrm{mg})$.

Data for 1-(1-Heptynyl)-4-nitrobenzene (3f):

bp: $\quad 155{ }^{\circ} \mathrm{C}(0.1 \mathrm{mmHg}, \mathrm{ABT})$ 
${ }^{1} \underline{\mathrm{H} \mathrm{NMR}}: \quad\left(500 \mathrm{MHz}, \mathrm{CHCl}_{3}\right)$

$8.15\left(\mathrm{dd}, J=2.5,8.5,2 \mathrm{H}, 2 \times \mathrm{HC}\left(3^{\prime}\right)\right), 7.50\left(\mathrm{dd}, J=2.5,7.5,2 \mathrm{H}, 2 \times \mathrm{HC}\left(2^{\prime}\right)\right)$, $2.42\left(\mathrm{t}, J=7.0,2 \mathrm{H}, \mathrm{H}_{2} \mathrm{C}(3)\right), 1.61$ (pent, $\left.J=7.0,2 \mathrm{H}, \mathrm{H}_{2} \mathrm{C}(4)\right), 1.34-1.45$ (m, $4 \mathrm{H}$, $\mathrm{H}_{2} \mathrm{C}(5$ and 6$), 0.93\left(\mathrm{t}, J=7.0,3 \mathrm{H}, \mathrm{H}_{3} \mathrm{C}(7)\right)$

${ }^{13} \underline{\mathrm{C} \mathrm{NMR}}: \quad\left(126 \mathrm{MHz}, \mathrm{CHCl}_{3}\right)$

$146.6\left(\mathrm{C}\left(4^{\prime}\right)\right), 132.3\left(\mathrm{C}\left(2^{\prime}\right)\right), 131.2\left(\mathrm{C}\left(1^{\prime}\right)\right), 123.5\left(\mathrm{C}\left(3^{\prime}\right)\right), 96.8(\mathrm{C}(1)), 79.3$ (C(2)), $31.1(\mathrm{C}(5)), 28.1(\mathrm{C}(4)), 22.2(\mathrm{C}(6)), 19.5(\mathrm{C}(3)), 13.9(\mathrm{C}(7))$

IR: (neat)

2932 (m), 2860 (m), 2360 (w), 2219(w), 1604 (s), 1594 (s), 1518 (s), 1342(s), $1107(\mathrm{~m}), 853(\mathrm{~s}), 750(\mathrm{~m})$

MS: $\quad(\mathrm{EI}, 70 \mathrm{ev})$

217 ( $\left.\mathrm{M}^{+}, 55\right), 202$ (30), 189 (18), 188 (100), 175 (12), 171 (12), 161 (12), 160 (16), 158 (28), 156 (18), 147 (18), 145 (11), 144 (14), 143 (19), 142 (78), 141 (40), 131 (18), 130 (28), 129 (49), 128 (80), 127 (24), 117 (19), 116 (64), 115 (65), 114 (45), 113 (23), 103 (12), 102 (36), 101 (14), 91 (17), 89 (19), 88 (22), 87 (12), 81 (38), 77 (20), 76 (13), 75 (19), 74 (10), 68 (17), 65 (10), 63 (30), 62 (13), $57(14), 55(41)$

TLC: $\quad R_{f} 0.42$ (hexane/EtOAc, 19/1) [silica gel, $\mathrm{KMnO}_{4}$ ]

GC: $\quad t_{\mathrm{R}} 10.88 \mathrm{~min}\left(\mathrm{HP}-5,15 \mathrm{psi}, 125{ }^{\circ} \mathrm{C}\right.$ for $4 \mathrm{~min}, 50{ }^{\circ} \mathrm{C} / \mathrm{min}$ to $\left.250{ }^{\circ} \mathrm{C}\right)$

Analysis: $\quad \mathrm{C}_{13} \mathrm{H}_{15} \mathrm{NO}_{2}(217.26)$

Calcd: C, 71.87; H, $6.96 \quad$ N, 6.45\%

Found: C, 71.65; H, $6.89 \quad$ N, 6.48\% 
Preparation of 1-[4-(1-Heptynyl)phenyl]ethanone (3g) (Table 2, entry 7)

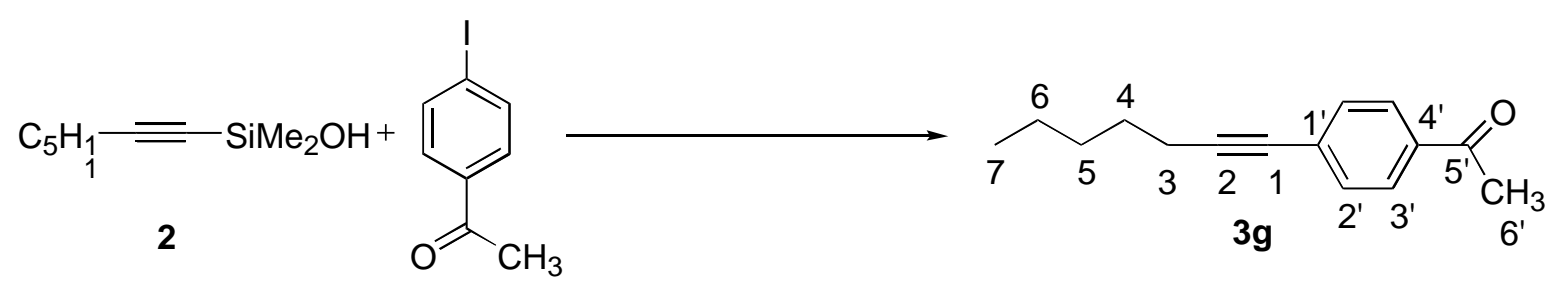

Following General Procedure II, 4-iodoacetophenone (246 mg, $1.00 \mathrm{mmol}$ ) was combined with potassium trimethylsilanolate $(256 \mathrm{mg}, 2.00 \mathrm{mmol}, 2.0$ equiv), copper(I) iodide (9.6 mg, $0.05 \mathrm{mmol}, 0.05$ equiv), $2 \mathrm{~mL}$ of DME, dimethyl(1-heptynyl)silanol (187 mg, 1.1 mmol, 1.1 equiv), and $\mathrm{PdCl}_{2}\left(\mathrm{PPh}_{3}\right)_{2}(17.2 \mathrm{mg}, 0.025 \mathrm{mmol}, 0.025$ equiv $)$. The product $\mathbf{3 g}$ was obtained as a colorless oil in $87 \%$ yield $(187 \mathrm{mg})$.

Data for 1-[4-(1-Heptynyl)phenyl]ethanone (3g):

bp: $\quad 155^{0} \mathrm{C}(1.0 \mathrm{mmHg}, \mathrm{ABT})$

${ }^{1} \underline{\mathrm{H} \mathrm{NMR}}: \quad\left(500 \mathrm{MHz}, \mathrm{CHCl}_{3}\right)$

$7.87\left(\mathrm{~d}, J=8.0,2 \mathrm{H}, 2 \times \mathrm{HC}\left(3^{\prime}\right)\right), 7.46\left(\mathrm{~d}, J=7.5,2 \mathrm{H}, 2\right.$ x $\left.\mathrm{HC}\left(2^{\prime}\right)\right), 2.58$ (s, $3 \mathrm{H}$, $\left.\mathrm{H}_{3} \mathrm{C}\left(6^{\prime}\right)\right), 2.42$ (t, $\left.J=7.0,2 \mathrm{H}, \mathrm{H}_{2} \mathrm{C}(3)\right), 1.61$ (pent, $\left.J=7.5,2 \mathrm{H}, \mathrm{H}_{2} \mathrm{C}(4)\right), 1.34-$ $1.45\left(\mathrm{~m}, 4 \mathrm{H}, \mathrm{H}_{2} \mathrm{C}\left(5\right.\right.$ and 6), $0.92\left(\mathrm{t}, J=7.0,3 \mathrm{H}, \mathrm{H}_{3} \mathrm{C}(7)\right)$

${ }^{13} \mathrm{C} \mathrm{NMR}: \quad\left(126 \mathrm{MHz}, \mathrm{CHCl}_{3}\right)$

$197.2\left(\mathrm{C}\left(5^{\prime}\right)\right), 135.4\left(\mathrm{C}\left(4^{\prime}\right)\right), 131.4\left(\mathrm{C}\left(2^{\prime}\right)\right), 128.9\left(\mathrm{C}\left(1^{\prime}\right)\right), 127.9\left(\mathrm{C}\left(3^{\prime}\right)\right), 94.2$ (C(1)), $79.8(\mathrm{C}(2)), 30.8(\mathrm{C}(5)), 28.0(\mathrm{C}(4)), 26.3\left(\mathrm{C}\left(6^{\prime}\right)\right), 21.9(\mathrm{C}(6)), 19.2$ $(\mathrm{C}(3)), 13.7(\mathrm{C}(7))$

IR: (neat) 3353 (m), 2933 (s), 2861 (s), 2360 (w), 2218 (m), 1927 (w), 1684 (s), 1601 (s), 1403(s), 1357 (s), 1263 (s), 1178 (m), 956 (s), 840 (s) 
MS: $\quad(E I, 70 \mathrm{ev})$

214 ( $\left.\mathrm{M}^{+}, 47\right), 200$ (17), 199 (100), 185 (30), 171 (15), 157 (36), 155 (11), 143

(33), 142 (31), 141 (13), 129 (61), 128 (32), 127 (20), 115 (39), 114 (46), 113

(17), 101 (11), 88 (14), 77 (12), 75 (11), 63 (17)

TLC: $\quad R_{f} 0.16$ (hexane/EtOAc, 19/1) [silica gel, $\mathrm{KMnO}_{4}$ ]

GC: $\quad t_{\mathrm{R}} 10.51 \mathrm{~min}\left(\mathrm{HP}-5,15 \mathrm{psi}, 125{ }^{\circ} \mathrm{C}\right.$ for $4 \mathrm{~min}, 50{ }^{\circ} \mathrm{C} / \mathrm{min}$ to $250{ }^{\circ} \mathrm{C}$

Analysis: $\quad \mathrm{C}_{15} \mathrm{H}_{18} \mathrm{O}(214.30)$

Calcd: $\quad$ C, 84.07; H, $8.47 \%$

Found: $\quad$ C, 84.21; H, $8.57 \%$

\section{Preparation of 3-(1-Heptynyl)benzenemethanol (3h) (Table 2, entry 8)}

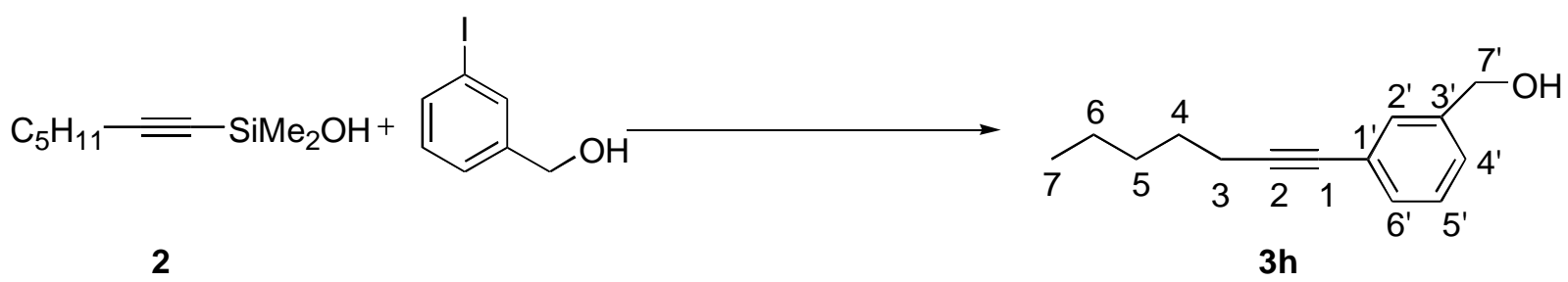

Following General Procedure II, 3-iodobenzyl alcohol (234 mg, $1.00 \mathrm{mmol}$ ) was combined with potassium trimethylsilanolate $(256 \mathrm{mg}, 2.00 \mathrm{mmol}, 2.0$ equiv), copper(I) iodide (9.6 mg, $0.05 \mathrm{mmol}, 0.05$ equiv), $2 \mathrm{~mL}$ of DME, dimethyl(1-heptynyl)silanol (187 mg, 1.1 mmol, 1.1 equiv), and $\mathrm{PdCl}_{2}\left(\mathrm{PPh}_{3}\right)_{2}(17.2 \mathrm{mg}, 0.025 \mathrm{mmol}, 0.025$ equiv $)$. The product $\mathbf{3 h}$ was obtained as a colorless oil in $84 \%$ yield $(169 \mathrm{mg})$.

Data for 3-(1-Heptynyl)benzenemethanol (3h):

bp: $\quad 140{ }^{0} \mathrm{C}(0.5 \mathrm{mmHg}, \mathrm{ABT})$ 


\section{${ }^{1} \underline{\mathrm{H} N M R}: \quad\left(500 \mathrm{MHz}, \mathrm{CHCl}_{3}\right)$}

$7.36\left(\mathrm{~s}, 1 \mathrm{H}, \mathrm{HC}\left(2^{\prime}\right)\right), 7.30\left(\mathrm{td}, J=1.5,7.01 \mathrm{H}, \mathrm{HC}\left(6^{\prime}\right)\right), 7.20-7.24(\mathrm{~m}, 2 \mathrm{H}$, $\left.\mathrm{HC}\left(5^{\prime}\right), \mathrm{HC}\left(4^{\prime}\right)\right), 4.59$ (d, $\left.J=4.0,2 \mathrm{H}, \mathrm{H}_{2} \mathrm{C}\left(7^{\prime}\right)\right), 2.38$ (t, $\left.J=7.0,2 \mathrm{H}, \mathrm{H}_{2} \mathrm{C}(3)\right), 2.22$ (d, $J=4.0,1 \mathrm{H},-\mathrm{OH}), 1.62$ (pent, $\left.J=7.0,2 \mathrm{H}, \mathrm{H}_{2} \mathrm{C}(4)\right), 1.32-1.44$ (m, $4 \mathrm{H}, \mathrm{H}_{2} \mathrm{C}(5$ and 6), $0.90\left(\mathrm{t}, J=7.5,3 \mathrm{H}, \mathrm{H}_{3} \mathrm{C}(7)\right)$

${ }^{13} \underline{\mathrm{C} \mathrm{NMR}}: \quad\left(126 \mathrm{MHz}, \mathrm{CHCl}_{3}\right)$

140.8 (C(1')), $130.7\left(\mathrm{C}\left(6^{\prime}\right)\right), 129.9$ (C(2’)), 128.4 (C(5')), 126.0 (C(4')), 124.2 (C(3’)), $\left.90.6(\mathrm{C}(1)), 80.3(\mathrm{C}(2)), 64.7 \mathrm{C}\left(7^{\prime}\right)\right), 31.0(\mathrm{C}(5)), 28.3(\mathrm{C}(4)), 22.2(\mathrm{C}(6))$, $19.3(\mathrm{C}(3)), 13.9(\mathrm{C}(7))$

IR: $\quad$ (neat)

3601 (m), 3448 (w,b), 2959 (s), 2861 (s), 2365 (w), 2228 (w), 1602 (m), 1483 (m), 1459 (m), 1431 (m), $1379(\mathrm{~m}), 1269$ (s), 1033 (m), 793 (s), 728 (s)

$\underline{\mathrm{MS}}: \quad(\mathrm{EI}, 70 \mathrm{ev})$

202 ( $\left.\mathrm{M}^{+}, 62\right), 173$ (32), 171 (13), 155 (18), 145 (61), 144 (11), 143 (62), 142

(16), 141 (15), 131 (16), 130 (13), 129 (88), 128 (52), 127 (17), 118 (11), 117

(100), $116(17), 115(65), 91(32), 77$ (15), 63 (12)

TLC: $\quad R_{f} 0.05$ (hexane/EtOAc, 19/1) [silica gel, $\mathrm{KMnO}_{4}$ ]

GC: $\quad t_{\mathrm{R}} 9.94 \mathrm{~min}\left(\mathrm{HP}-5,15 \mathrm{psi}, 125{ }^{\circ} \mathrm{C}\right.$ for $4 \mathrm{~min}, 50{ }^{0} \mathrm{C} / \mathrm{min}$ to $\left.250{ }^{\circ} \mathrm{C}\right)$

Analysis: $\quad \mathrm{C}_{14} \mathrm{H}_{18} \mathrm{O}(202.29)$

Calcd: $\quad$ C, 83.12; $\quad \mathrm{H}, 8.97 \%$

Found: $\quad$ C, 83.08; H, $9.27 \%$ 
Preparation of 1-(1-Heptynyl)-2-methoxybenzene (3i) ${ }^{\text {Table } 2 \text {, entry 9) }}{ }^{1}$
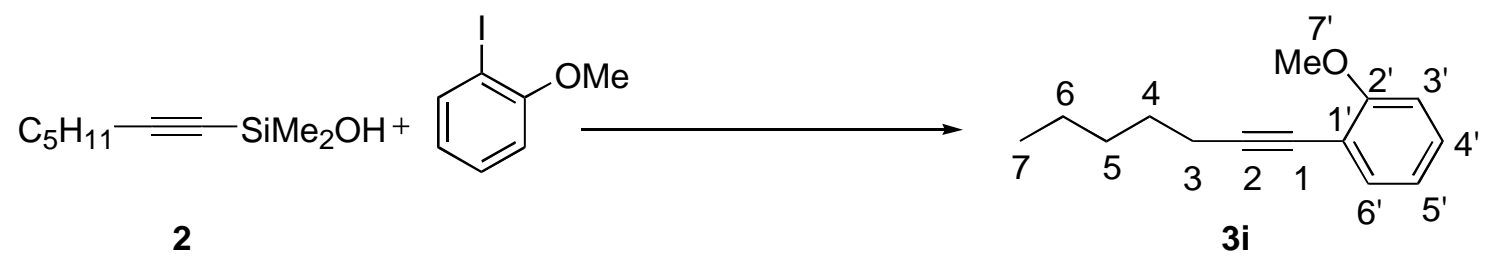

Following General Procedure II, 2-iodoanisole (234 mg, $1.00 \mathrm{mmol}$ ) was combined with potassium trimethylsilanolate (256 $\mathrm{mg}, 2.00 \mathrm{mmol}, 2.0$ equiv), copper(I) iodide (9.6 mg, 0.05 mmol, 0.05 equiv), $2 \mathrm{~mL}$ of DME, dimethyl(1-heptynyl)silanol (187 mg, $1.1 \mathrm{mmol}, 1.1 \mathrm{equiv})$, and $\mathrm{PdCl}_{2}\left(\mathrm{PPh}_{3}\right)_{2}(17.2 \mathrm{mg}, 0.025 \mathrm{mmol}, 0.025$ equiv). The product $3 \mathbf{i}$ was obtained as a colorless oil in $93 \%$ yield $(188 \mathrm{mg})$.

Data for 1-(1-Heptynyl)-2-methoxybenzene (3i):

bp: $\quad 115{ }^{0} \mathrm{C}(0.5 \mathrm{mmHg}, \mathrm{ABT})$

${ }^{1}$ H NMR: $\quad\left(500 \mathrm{MHz}, \mathrm{CHCl}_{3}\right)$

$7.38\left(\mathrm{dd}, J=2.0,7.5,1 \mathrm{H}, \mathrm{HC}\left(5^{\prime}\right)\right), 7.23\left(\mathrm{td}, J=2.0,7.51 \mathrm{H}, \mathrm{HC}\left(4^{\prime}\right)\right), 6.84-6.89$

(m, $\left.2 \mathrm{H}, \mathrm{HC}\left(3^{\prime}\right), \mathrm{HC}\left(6^{\prime}\right)\right), 3.88$ (s, $\left.3 \mathrm{H}, \mathrm{H}_{3} \mathrm{C}\left(7^{\prime}\right)\right), 2.46\left(\mathrm{t}, J=7.0,2 \mathrm{H}, \mathrm{H}_{2} \mathrm{C}(3)\right)$, 1.62 (pent, $\left.J=7.5,2 \mathrm{H}, \mathrm{H}_{2} \mathrm{C}(4)\right), 1.43$ (pent, $J=7.5,2 \mathrm{H}, \mathrm{H}_{2} \mathrm{C}(5)$ ), 1.37 (pent, $J$ $\left.=7.0,2 \mathrm{H}, \mathrm{H}_{2} \mathrm{C}(6)\right), 0.92\left(\mathrm{t}, J=7.0,3 \mathrm{H}, \mathrm{H}_{3} \mathrm{C}(7)\right)$

${ }^{13} \mathrm{C} \mathrm{NMR}: \quad\left(126 \mathrm{MHz}, \mathrm{CHCl}_{3}\right)$

$159.6\left(\mathrm{C}\left(6^{\prime}\right)\right), 133.4\left(\mathrm{C}\left(2^{\prime}\right)\right), 128.6\left(\mathrm{C}\left(4^{\prime}\right)\right), 120.1\left(\mathrm{C}\left(5^{\prime}\right)\right), 112.9\left(\mathrm{C}\left(1^{\prime}\right)\right), 110.3$ (C(3')), $94.5(\mathrm{C}(1)), 76.3(\mathrm{C}(2)), 55.5\left(\mathrm{C}\left(7^{\prime}\right)\right) 30.9(\mathrm{C}(5)), 28.3(\mathrm{C}(4)), 22.0$ (C(6)), $19.5(\mathrm{C}(3)), 13.7(\mathrm{C}(7))$ 
IR: $\quad$ (neat)

2932 (m), 2859 (m), 1595 (m), 1575 (m), 1492 (s), 1464 (m), 1433 (m), 1261 (s),

$1117(\mathrm{~m}), 1026(\mathrm{~m}), 750(\mathrm{~s})$

MS: $\quad(\mathrm{EI}, 70 \mathrm{ev})$

$202\left(\mathrm{M}^{+}, 97\right), 187$ (14), 173 (45), 160 (16), 159 (38), 158 (23), 148 (13), 147

(100), 145 (35), 144 (19), 134 (12), 132 (16), 131 (58), 129 (22), 128 (2), 127

(16), 121 (23), 119 (11), 117 (26), 116 (20), 115 (78), 107 (15), 103 (10), 102

(20), 91 (61) 89 (19), 77 (19), 76 (12), 75 (12), 65 (11), 63 (16)

TLC: $\quad R_{f} 0.31$ (hexane/EtOAc, 19/1) [silica gel, $\mathrm{KMnO}_{4}$ ]

GC: $\quad t_{\mathrm{R}} 9.47 \mathrm{~min}\left(\mathrm{HP}-5,15 \mathrm{psi}, 125{ }^{\circ} \mathrm{C}\right.$ for $4 \mathrm{~min}, 50{ }^{\circ} \mathrm{C} / \mathrm{min}$ to $\left.250{ }^{\circ} \mathrm{C}\right)$

Analysis: $\quad \mathrm{C}_{14} \mathrm{H}_{18} \mathrm{O}(202.29)$

Calcd: $\quad$ C, 83.12; $\quad \mathrm{H}, 8.97 \%$

Found: $\quad$ C, 83.19; $\quad$ H, 8.93\%

\section{Preparation of 1-(1-Heptynyl)-2-methylbenzene (3j) (Table 2, entry 10)}

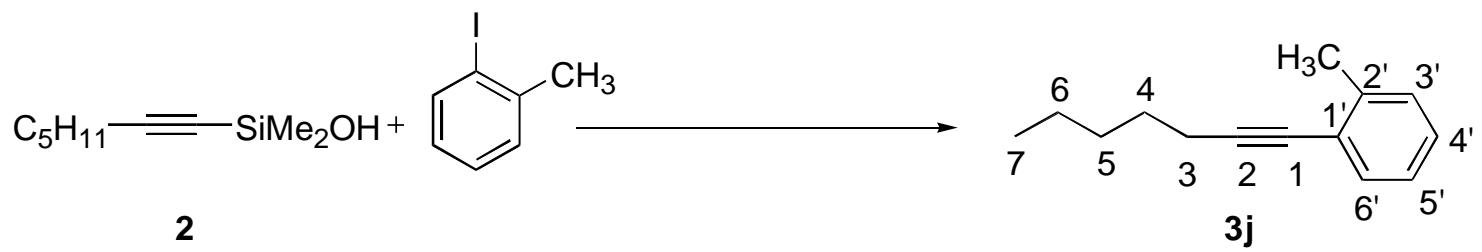

Following General Procedure II, 2-iodotoluene $(218 \mathrm{mg}, 1.00 \mathrm{mmol})$ was combined with potassium trimethylsilanolate (256 mg, $2.00 \mathrm{mmol}, 2.0$ equiv), copper(I) iodide (9.6 mg, 0.05 mmol, 0.05 equiv), $2 \mathrm{~mL}$ of DME, dimethyl(1-heptynyl)silanol (187 mg, $1.1 \mathrm{mmol}, 1.1 \mathrm{equiv})$, and $\mathrm{PdCl}_{2}\left(\mathrm{PPh}_{3}\right)_{2}(17.2 \mathrm{mg}, 0.025 \mathrm{mmol}, 0.025$ equiv). The product $\mathbf{3 j}$ was obtained as a colorless oil in $92 \%$ yield $(171 \mathrm{mg})$. 
Data for 1-(1-Heptynyl)-2-methylbenzene (3j):

bp: $\quad 115{ }^{0} \mathrm{C}(0.5 \mathrm{~mm} \mathrm{Hg} \mathrm{ABT})$

${ }^{1} \underline{\mathrm{H} \text { NMR}}: \quad\left(500 \mathrm{MHz}, \mathrm{CHCl}_{3}\right)$

$7.36\left(\mathrm{~d}, J=7.5,1 \mathrm{H}, \mathrm{HC}\left(6^{\prime}\right)\right), 7.17-7.18$ (m, $\left.2 \mathrm{H}, \mathrm{HC}\left(3^{\prime}\right), \mathrm{HC}\left(5^{\prime}\right)\right), 7.10-7.13$ (m, $\left.1 \mathrm{H}, \mathrm{HC}\left(4^{\prime}\right)\right), 2.46$ (t, $\left.J=7.0,2 \mathrm{H}, \mathrm{H}_{2} \mathrm{C}(3)\right), 2.43$ (s, $\left.3 \mathrm{H},-\mathrm{CH}_{3}\right), 1.62$ (pent, $J=$ 7.5, $2 \mathrm{H}, \mathrm{H}_{2} \mathrm{C}(4)$ ), 1.44 (pent, $J=6.5,2 \mathrm{H}, \mathrm{H}_{2} \mathrm{C}(5)$ ), 1.38 (pent, $J=6.5,2 \mathrm{H}$, $\left.\mathrm{H}_{2} \mathrm{C}(6)\right), 0.92\left(\mathrm{t}, J=7.5,3 \mathrm{H}, \mathrm{H}_{3} \mathrm{C}(7)\right)$

${ }^{13}$ C NMR: $\quad\left(126 \mathrm{MHz}, \mathrm{CHCl}_{3}\right)$

$139.7\left(\mathrm{C}\left(6^{\prime}\right)\right), 131.5\left(\mathrm{C}\left(2^{\prime}\right)\right), 129.0\left(\mathrm{C}\left(5^{\prime}\right)\right), 127.2\left(\mathrm{C}\left(4^{\prime}\right)\right), 125.1\left(\mathrm{C}\left(3^{\prime}\right)\right), 123.6$ (C(1')), $94.2(\mathrm{C}(1)), 79.1(\mathrm{C}(2)), 30.8(\mathrm{C}(5)), 28.3(\mathrm{C}(4)), 22.0(\mathrm{C}(6)), 20.4$ ($\left.\mathrm{CH}_{3}\right), 19.2(\mathrm{C}(3)), 13.7(\mathrm{C}(7))$

IR: (neat)

2957 (s), 2932 (s), 2860 (s), 2234 (w), 1912 (w), 1600 (s), 1486 (s), 1456 (s), $1378(\mathrm{~m}), 1330(\mathrm{~m}), 1044(\mathrm{~m}), 755$ (s)

MS: $\quad(\mathrm{EI}, 70 \mathrm{ev})$

186 ( M+ 96), 171 (11), 158 (11), 157 (62), 144 (13), 143 (34), 142 (27), 141

(18), 132 (10), 131 (88), 130 (15), 129 (100), 128 (93), 127 (28), 116 (15), 115

(39), 105 (18), $91(15)$

TLC: $\quad R_{f} 0.55$ (hexane/EtOAc, 19/1) [silica gel, $\mathrm{KMnO}_{4}$ ]

GC: $\quad t_{\mathrm{R}} 8.67 \mathrm{~min}\left(\mathrm{HP}-5,15 \mathrm{psi}, 125{ }^{0} \mathrm{C}\right.$ for $4 \mathrm{~min}, 50{ }^{0} \mathrm{C} / \mathrm{min}$ to $\left.250{ }^{\circ} \mathrm{C}\right)$

Analysis: $\quad \mathrm{C}_{14} \mathrm{H}_{18}(186.29)$

Calcd: C, 90.26; H, $9.74 \%$

Found: $\quad$ C, 89.97; H, $9.82 \%$ 
Preparation of 1-(1-Heptynyl)-4-methoxybenzene (3a) with Isolation of 1,1'-(1,4-Dipentyl-

\section{1,2,3-butatriene-1,4-diyl)bis[4-methoxybenzene] (4) and 1,1'-[1-Pentyl-2-(1-heptynyl)-1,2-} ethenediyl]bis[4-methoxybenzene] (5)
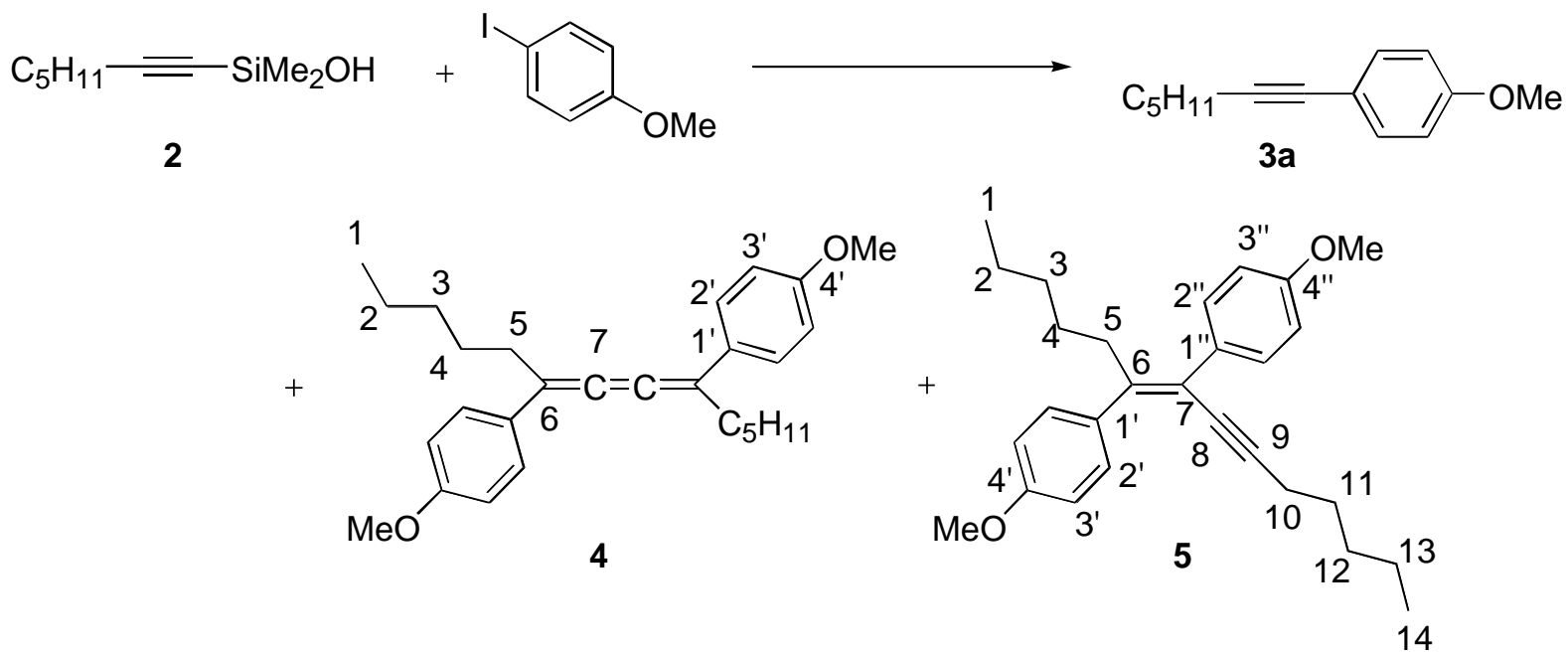

To an oven-dried, 25-mL, round-bottom flask equipped with a magnetic stir bar was added $512 \mathrm{mg}$ (4.0 mmol, 4.0 equiv) of potassium trimethylsilanolate under a dry nitrogen atmosphere. To this was added sequentially $4 \mathrm{~mL}$ of DME, dimethyl(1-heptynyl)silanol (375 mg, 2.2 mmol, 1.1 equiv), 4-iodoanisole (468 mg, $2.0 \mathrm{mmol})$, and $\mathrm{PdCl}_{2}\left(\mathrm{PPh}_{3}\right)_{2}(34.4 \mathrm{mg}, 0.05$ mmol, 0.025 equiv). The resulting solution was stirred at room temperature for $8 \mathrm{~h}$. The reaction mixture was taken up in $15 \mathrm{~mL}$ of $\mathrm{Et}_{2} \mathrm{O}$. The combined organic phases were washed with water $(2 \times 15 \mathrm{~mL})$ and the aqueous phase was back extracted with $\mathrm{Et}_{2} \mathrm{O}(2 \times 15 \mathrm{~mL})$. The combined organic extracts were washed with brine $(15 \mathrm{~mL})$ and the were dried over $\mathrm{MgSO}_{4}$. The volatile solvents were removed in vacuo to provide a dark oil. The oil was taken up in $5 \mathrm{~mL}$ of $\mathrm{Et}_{2} \mathrm{O}$ and loaded onto a $30 \mathrm{~mm}$ x $40 \mathrm{~cm}$ silica gel column which was eluted with pentane/ $\mathrm{Et}_{2} \mathrm{O}$ ), 50/1, (5 psi) to afford 3a (109 mg, 59\%) as a pale yellow along with a yellow semisolid. The semisolid was recrystallized from $3 \mathrm{~mL}$ of hot ethanol to give $29 \mathrm{mg}$ (7\%) of 4 as a bright- 
yellow, crystalline solid. The mother liquor was concentrated in vacuo to remove the volatile solvents. The resulting oil was purified by preparative TLC with pentane/Et ${ }_{2} \mathrm{O}, 20 / 1$ to give 15 mg (4\%) of 5 as a colorless oil.

\section{Data for 4:}

${ }^{1} \underline{\mathrm{H} \text { NMR}}: \quad\left(500 \mathrm{MHz}, \mathrm{CHCl}_{3}\right)$

$7.57\left(\mathrm{dd}, J=2.5,8.5,4 \mathrm{H}, 4 \times \mathrm{HC}\left(2^{\prime}\right)\right), 6.88\left(\mathrm{dd}, J=2.5,8.5,4 \mathrm{H}, 4 \times \mathrm{HC}\left(1^{\prime}\right)\right)$, $3.83\left(\mathrm{~s}, 6 \mathrm{H}, 2 \mathrm{x}-\mathrm{OCH}_{3}\right), 2.67$ (t, $\left.J=9.5,4 \mathrm{H}, 2 \times \mathrm{H}_{2} \mathrm{C}(5)\right), 1.79$ (pent, $J=9.5,4$ $\left.\mathrm{H}, 2 \times \mathrm{H}_{2} \mathrm{C}(4)\right), 1.40-1.54\left(\mathrm{~m}, 8 \mathrm{H}, 4 \times \mathrm{H}_{2} \mathrm{C}(2,3)\right), 0.93\left(\mathrm{t}, J=9,6 \mathrm{H}, 2 \times \mathrm{H}_{3} \mathrm{C}(1)\right)$ ${ }^{13} \mathrm{C} \mathrm{NMR}: \quad\left(126 \mathrm{MHz}, \mathrm{CHCl}_{3}\right)$

$158.84\left(\mathrm{C}\left(4^{\prime}\right)\right), 151.35(\mathrm{C}(7)), 132.00\left(\mathrm{C}\left(2^{\prime}\right)\right), 127.42\left(\mathrm{C}\left(1^{\prime}\right)\right), 117.47\left(\mathrm{C}\left(3^{\prime}\right)\right)$, $113.79(\mathrm{C}(6)), 55.32$ (-OMe), $33.27(\mathrm{C}(5)), 31.73(\mathrm{C}(4)), 28.56(\mathrm{C}(3)), 22.67$ $(\mathrm{C}(2)), 14.03,(\mathrm{C}(1))$

MS: (EI, $70 \mathrm{eV})$

$404\left(\mathrm{M}^{+}, 100\right), 347(22), 277(13), 121(22)$

Data for 5:

${ }^{1} \underline{\mathrm{H} \mathrm{NMR}}: \quad\left(500 \mathrm{MHz}, \mathrm{CHCl}_{3}\right)$

$7.43(\mathrm{~d}, J=8.5,2 \mathrm{H}, 2$ x HC(3') ) ), 7.30 (d, $J=9,2 \mathrm{H}, 2$ x HC(3')), 6.89 (m, $4 \mathrm{H}$ $2 \times\left(\mathrm{HC}\left(2^{\prime}, 2^{\prime}\right.\right.$ ') ), 3.83 (s, $6 \mathrm{H}, 2$ x -OMe), 2.40 (t, $\left.J=7.5,2 \mathrm{H}, \mathrm{H}_{2} \mathrm{C}(10)\right), 2.14$ (t, $\left.J=7,2 \mathrm{H}, \mathrm{H}_{2} \mathrm{C}(5)\right), 1.11-1.34\left(\mathrm{~m}, 12 \mathrm{H}, \mathrm{H}_{2} \mathrm{C}(2,3,4,11,12,13), 0.83(\mathrm{t}, J=7,3 \mathrm{H}\right.$, $\left.\mathrm{H}_{3} \mathrm{C}(14)\right), 0.77\left(\mathrm{t}, J=7,3 \mathrm{H}, \mathrm{H}_{3} \mathrm{C}(1)\right)$

${ }^{13} \underline{\mathrm{C} \mathrm{NMR}}: \quad\left(126 \mathrm{MHz}, \mathrm{CHCl}_{3}\right)$

158.49 (C(4')), 158.41 (C(4'’)), 147.69 (C(6)), 134.27 (C(1')), $133.16\left(\mathrm{C}\left(1^{\prime \prime}\right)\right)$, $129.92\left(\mathrm{C}\left(2^{\prime}\right)\right), 129.79\left(\mathrm{C}\left(2^{\prime \prime}\right)\right), 119.91(\mathrm{C}(7)), 113.47\left(\mathrm{C}\left(3^{\prime}\right)\right), 112.99\left(\mathrm{C}\left(3^{\prime \prime}\right)\right)$, 
$92.53(\mathrm{C}(9)), 82.48(\mathrm{C}(8)), 55.23$ (OMe), 55.12 (OMe), 33.81 (C(10)), 31.60

(C(5)), 30.86(C(11)), $28.36(\mathrm{C}(4)), 28.22(\mathrm{C}(12)), 22.26(\mathrm{C}(3)), 22.19(\mathrm{C}(13))$, 19.56(C(2)), $13.93(\mathrm{C}(14)), 13.91(\mathrm{C}(1))$

MS: $\quad(E I, 70 \mathrm{eV})$

$404\left(\mathrm{M}^{+}, 100\right), 347(24), 277(9)$

\section{References}

(1) Chapdelain, M.; Warwick, P.; Shaw, A. J. Org. Chem. 1989, 54, 1218.

(2) Okuro, K.; Furuune, M.; Enna, M.; Miura, M.; Nomura, M. J. Org. Chem. 1993, $58,4716$. 